\title{
The Fundamental and Universal Nature of Boltzmann's Constant
}

\author{
L.C. Biedenharn ${ }^{1}$ \\ Center for Particle Physics, Department of Physics, University of Texas at Austin, \\ Austin, TX 78712-1081 \\ and \\ J.C. Solem \\ Theoretical Division, Los Alamos National Laboratory, Los Alamos, NM 87545
}

\section{Dedication:}

This paper is dedicated to Professor Paulo Leal Ferreira, as a contribution to celcbrate the occasion of his seventieth birthday.

\begin{abstract}
The nature of Boltzmann's constant is very unclear in the physics literature. In the first part of this paper, on general considerations, we examine this situation in detail and demonstrate the conclusion that Boltzmann's constant is indeed both fundamental and universal. As a consequence of our development we find there is an important implication of this work for the problem of the entropy of information. In the second part we discuss, Szilard's famous construction showing in detail how his result is incompatible with the demonstrations in both parts I and II.
\end{abstract}

1. General Considerations

\subsection{Introduction}

Of all the fundamental constants of physics Boltzmann's constant, (and its nature) is the most unclear in the physics literature. Generally, but not always, Boltzmann's constant is accorded fundamental status, as for example in the most recent compilation of "The Fundamental Physical Constants" by Cohen and Taylor [1].

In a 1983 article, Weinberg [2] addressed the question as to "what is a fundamental constant?" His answer, in part, included these comments:

"The list of fundamental constants depends on who (and I would add on when one) is compiling the list... A hydrodynamicist would put in the list the density and viscosity of water, an atomic physicist would put the mass of the proton and the charge of the electron."

Weinberg's definition of a list of fundamental constants is this:

"A list of constants whose value we cannot calculate in terms of more fundamental constants, not just because the calculation is too hard, but because we do not know of anything more fundamental ..."

\footnotetext{
${ }^{1}$ Consultant: Theoretical Division, Los Alamos National Laboratory, Los Alamos, NM 87545

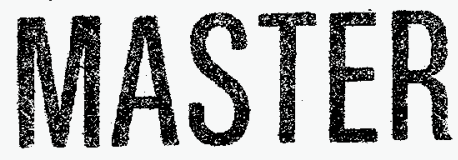


Weinberg concludes by saying:

"The membership of such a list thus reflects our present understanding of fundamental physics."

Veneziano [3] returned to the question of fundamental constants in 1992, and gave examples of fundamental constants (among them $\hbar$ and $c$ ) and then mentioned Boltzmann's constant with this comment:

"As far as Boltzmann's constant $k$ is concerned, I hope we all agree on refusing it membership! Had we understood immediately that temperature is randomly distributed energy, we would have measured it in ergs without any need of introducing $k$. What is fundamental is not $k$, but the identification of temperature with randomly distributed kinetic energy. In fact, in modern textbooks, temperature is presented from the very beginning as an energy."

(We comment on this view below.)

Actually with dimensional constants, fundamental or not, things are not quite so simple, and there are extreme views which have been advanced in many textbooks [4].

Just to cite an example, Brian Ellis [4] concludes a chapter as follows:

"Simply by adopting different conventions concerning the expression of physical laws, universal (dimensionful) constants may be created or eliminated at will. It is quite absurd to suppose that these constants represent magnitudes of peculiar invariant properties of space and matter."

Thus, indeed, some people would like to go as far as saying that there is no meaning to a dimensionful fundamental constant! They should all, it is claimed, be taken out of the list of fundamental constants.

Such a viewpoint is actually much too extreme, as can be seen in this way. A dimensional relation between two physical quantities has only three invariant possible values for the dimensional constant: zero, finite or infinite. The important statement is that a finite value exists, which can be a fact of crucial physical importance.

The point made by Veneziano, in excluding Boltzmann's constant as fundamental, is a very common view and requires some discussion. The crucial point is that the thermal energy (of a distribution) is not an ordinary energy (say like the kinetic energy of an electron) but is a very special energy (not assignable to an elementary particle). This is clear, as Max Born points out [5], from the fact that thermal energy obeys a first-order equation in time, and hence thermal energy has no inertia. (In fact, the basic problem presented by thermal energy, was to distinguish heat energy $(\Delta U)$, which is conserved (by the First Law of Thermodynamics) from the "caloric energy" (entropy) which is not conserved (by the Second Law of Thermodynamics).) The vast difference between the nature of these two distinct "thermal energies" is clear from the fact that the First Law is absolute, whereas the Second Law is statistical.

It is our aim to discuss the nature of Boltzmann's constant from several points of view, in order to make clear the essential reason why Boltzmann's constant is, in our view, both fundamental and universal. To do so, will require first that we review 


\section{DISCLAIMER}

Portions of this document may be illegible in electronic image products. Images are produced from the best available original document. 

$(\S 1.2)$ some basic results from (classical) thermodynamics, as amended in critical ways by quantum mechanics. We then turn $(\S 1.3)$ to a paper by Koppe and Huber [6], which demonstrates some remarkable results, calculating ab initio Boltzmann's constant! This work is then analyzed in detail to show that it is not as simple as it purports to be, presenting our analysis in $§ 1.4$.

\subsection{Absolute Temperature}

Temperature is an intuitive concept because it invades our everyday experience. It follows in the Aristotelian tradition of appealing to common knowledge, much as the concepts of force, mass, and time appeal to common knowledge, each being fraught with difficulties when examined in greater detail. At the most primitive level, temperature is an ordered sequence of points marking common levels of thermodynamic equilibrium, and the empirical fact that equilibrium will be approached by most systems is sometimes referred to as the zeroth law of thermodynamics [7]. (We remark that this law would be invalid without the existence of quantum mechanics, so that classical thermodynamics is not an a priori self-contained discipline.) An alternative statement is that temperature determines the direction of heat flow when an object is placed in contact with another object. To be pleasantly circular, heat is then defined as energy in transit due to temperature difference - again appealing to intuition to subdue objections. The definition provides order but no measure, and physics thrives on numbers.

In order to get a number, we appeal to the less intuitive concept of entropy. Entropy has entered popular usage as a measure of disorder, and it is common knowledge that systems set in order inevitably become disordered in the course of time. Few people know how to assign a number to disorder, whereas numerical temperature is used every day. A meaningful measure of disorder is $\log W$, where $W$ is the number of microscopic states corresponding to the state of the system. The thermodynamic definition adds a scale factor,

$$
\text { Entropy }=S=-k \log W,
$$

where $k \simeq 1.380662 \times 10^{-16} \mathrm{erg} \cdot{ }^{\circ} K^{-1}$ is Boltzmann's constant. The definition of temperature thereby becomes a well defined number,

$$
\frac{1}{T}=\frac{d S}{d Q}
$$

where $Q$ is heat. This is also the succinct statement of the second law [3].

\subsection{The Ab Initio Calculation of Boltzmann's Constant by Koppe and Huber}

Koppe and Huber introduce their paper [6] by citing in the first section an actual dialogue with other physicists concerning their calculation:

"Theoretician: By the way, it appears not to be generally known that Boltzmann's constant is not a fundamental constant of nature, but can be calculated from other, known, constants. 
Experimenter: So? What does that do for us then?

Theoretician: Unfortunately there exists not the slightest prospect to actually carry out the calculation.

Experimenter: ?

Theoretician: That is simply the result of the preposterous way in which the experimentalists have defined the Kelvin degree."

This surprising claim by Koppe and Huber would seem to settle, definitively, the nature of Boltzmann's constant. Certainly from the criterion of Weinberg (in $\S 1$ ), if their claim is correct, then Boltzmann's constant can hardly be considered fundamental.

How do Koppe and Huber actually carry out their calculation? First they notice that, quantum mechanically, the specific heat of the diatomic gas $H D$ shows a maximum at a definite measurable value of the Kelvin temperature. They then use the best available data on the interatomic interaction potential of $H$ and $D$, to calculate, quantum mechanically, the absolute value of the relative kinetic energy at which this maximum should occur.

Then by equating the two energies - the calculated energy for the maximum and the observed Kelvin temperature (measured experimentally) - they deduce a numerical value for the (dimensional) Boltzmann constant.

Remarkably they obtain a value for that Boltzmann constant that is in error by only $\approx 7 \%$.

It would appear from this calculation, that Koppe and Huber have certainly verified their claim. We examine this critically in the next section.

\subsection{Further Discussion}

The calculation, reviewed in $\S 1.3$, is eminently satisfying for Koppe and Huber demonstrate explicitly that a reasonable value of Boltzmann's constant $k$ can be calculated $a b$ initio using the absolute temperature scale.

Yet on further reflection, the Koppe/Huber calculation has its own puzzling aspects. The input datum for this calculation was the actual, measured, interaction potential between the two atoms, $\mathrm{H}$ and $\mathrm{D}$, of the $\mathrm{HD}$ molecule. (In principle, this interaction could itself be calculated $a b$ initio from the fundamental electromagnetic interaction of the $\mathrm{H}$ and $\mathrm{D}$ atoms.) What would have happened if they had used another pair of atoms, with very likely a quite different potential? Molecular interactions are, in the final analysis, electromagnetic; what if the calculations were necessarily to be at very high energies. Would the running (renormalized) value of the electromagnetic interaction then enter? Would Boltzmann's constant then itself have a "running" value? Or, to pick an extreme case, suppose we were to use baryons as composite objects in an extremely energetic thermal equilibrium, would Boltzmann's constant be the same for such a calculation using QCD? Conceivably the Boltzmann constant could be different for different interactions, and the accepted value of Boltzmann's constant in present day use would simply be a reflection of the fact that only electromagnetic interactions are of importance in everyday terrestrial physics. More mundanely, it is known that besides the famous triple point of water 
defining the Kelvin scale there are other triple points (in water) - why should we expect the calculations for these, in principle, to yield precisely the same value of Boltzmann's constant? Why, in other words, is Boltzmann's constant universal - if, indeed, it really is?

The answer to these questions can be found by examining, in more detail, the underlying physical assumptions and physical methodology implicit in the Koppe/Huber calculation. First of all the underlying physical calculation was necessarily quantum mechanical and it is essential to realize that the use of quantum mechanics, far from being an extension of classical thermodynamics and statistical mechanics, is actually necessary for these disciplines to be physically valid. Next one sees that the physical distribution functions of the rotational degrees of freedom (including the proper (quantal) "freezing-out" of the higher levels) were assumed to lead to thermal equilibrium with the translational degrees of freedom of the HD molecule (moving effectively as a point mass). These translational degrees of freedom, in effect, furnish the necessary "heat bath" to allow, by collisional interactions, the assumed thermal equilibrium of the rotational and translational degrees of freedom to be obtained.

The truly essential ingredient in the Koppe/Huber calculation can be seen to be the assumption of thermal equilibrium for the rotational and translational degrees of freedom. The calculated maximum in the specific heat curve played only the rôle of an identifying characteristic for determining a definite point on the absolute temperature scale with its associated, calculated, energy.

We argue that the universality of the Boltzmann constant stems from this requirement of thermal equilibrium (the "zeroth law" [7]).

An example (involving the same logic) might help here. Consider the situation for Kirchhoff's observation that different physical objects in a Hohlraum, at an elevated temperature, all radiate in precisely the same way and are, hence, indistinguishable. This is so despite the fact that the various objects may have, and do have, vastly different emissivities at different frequencies. It is the condition of thermal equilibrium that ensures the univerality found by Kirchhoff in a Hohlraum.

In exactly the same way, despite the multiplicity of possible interactions, characteristic quantal energies, and distinct quantal distribution functions, the condition of equilibrium implies that the Boltzmann constant is universal.

We conclude that Boltzmann's constant is indeed a fundamental, universal, constant.

\subsection{Application}

Our discussion began with the unusual rôle of Boltzmann's constant in the pantheon of fundamental (dimensional) constants, then used the little-known fact that this constant could be calculated $a b$ initio, and finally came, full-circle, to the conclusion that — despite all these particularities - Boltzmann's constant is nonetheless universal and fundamental. Several colleagues, with whom we have discussed these results, (to their initial disbelief), end with the put-down: "So what?" Aside from the satisfaction of deepened understanding, and, of course, pedagogical usefulness, is there anything more to these considerations? We think there is, and offer the following application as evidence.

The essential point made evident by our analysis is the fundamental rôle of ther- 
mal equilibrium in the determination not only of the absolute temperature but of Boltzmann's constant itself. Thermal equilibrium between different degrees of freedom can, however, only be achieved if there is some physical interaction between these different modes. What kind of interaction - QED, QCD, contact, whatever makes little difference, but some interaction there must be.

It follows that if there is no conceivable mechanism of interaction, there can be no thermal equilibrium, no meaning to temperature, and in consequence no meaning to "Boltzmann's constant" for such systems.

This conclusion is implied by our analysis, and, although this result sounds simultaneously obvious and vacuous, there is indeed an application.

Consider the famous (but not entirely uncontroversial) result of Szilard [8] who devised, and analyzed, a conceptual model for Maxwell's demon concluding that - to avoid violating the second law of thermodynamics - pure information must be associated with physical entropy. Szilard found that one bit of information was equivalent to a physical entropy of, at least, the amount: $k \log 2$. That is:

$$
S_{\text {per bit of information }} \geq k \log 2 .
$$

To arrive at this result, Szilard (just as in Einstein's analysis of Brownian motion) reversed Boltzmann's definition of entropy: $S=-k \log W$ (where $W$ is a probability). Szilard defined his probability as: $W_{1}=e^{-S_{1} / k}$. His result then followed for a twostate system using $W_{1}+W_{2} \leq 1$. To maintain the validity of the second law, Szilard postulated that the entropy deficit was to be made up by the "informational entropy."

This ingenious, and plausible, new concept found by Szilard - although attacked immediately and subsequently - has, to a surprising extent been accepted and extended by many physicists and mathematicians [9].

Let us consider Szilard's proposal in the light of our analysis of the rôle of Boltzmann's constant. We found, put succinctly, that if there is no physical interaction there is no Boltzmann's constant (that is, $k$ is undefined). So where does the constant $k$ in Szilard's basic formula, (eq. 3), come from? It can not be the universal Boltzmann $k$, since this requires that the "informational degrees of freedom" interact with physical degrees of freedom to achieve thermal equilibrium. Then, and only then, can a Boltzmann constant be defined for the informational degrees of freedom.

Information, per se, is, however, an idealized concept, which is in principle completely subjective, since the idealization necessarily eliminates information as such from the physical realm. Let us be precise: "information" is always carried by some physical object (be it computer memories, neuronal memories, or library pages, ....) and, as such, certainly has physical entropy. But this physical entropy is devicedependent, and varies by many orders of magnitude from one device to another. Informational entropy is, by contrast, the entropy associated with an idealized, deviceindependent, information structure (arrangement), with such an entropy being associated to the information itself and not to its physical carrier.

How is one to determine this purely informational entropy? The idealization involved in this concept seems to indicate minimal, or even vanishing, physical entropy. But how can one be sure?

Consider Szilard's formula again: $S_{\text {per bit of information }} \geq k \log 2$. Recall that $k$ is well-defined only in thermal equilibrium. What thermal equilibrium was involved in 
getting Szilard's result? Clearly the absolute temperature involved was irrelevant to his argument, and indeed his result - as given in eq. (3) - is manifestly independent of temperature. This leads to a contradiction.

According to the third law of thermodynamics (called the Nernst theorem by Fermi [10]), Szilard's result cannot be correct, since the third law asserts that: any physical entropy must vanish as $T \rightarrow 0$.

Remark: The third law has been itself the subject of controversy. The discussion by Fermi [10] and the very careful foundational analysis of Tisza [7], we believe, can resolve all of these objections. For the reader's confidence let us remark that the ideal gas law also violates the third law, but the quantum-mechanical ideal gas law for Fermi-Dirac (and Bose-Einstein) statistics verifies this law.

There is a way to escape the contradiction: take $k=0$ in eq. (3). This result that the "Boltzmann constant for information" is zero - is actually quite reasonable, since this is exactly what one might expect from our earlier analysis on the essential rôle of interactions, equilibrium and dimensional constants.

We conclude: idealized information is, from the idealization itself, devoid of physical interactions and in consequence has no physical entropy.

Remark: To avoid a possible misunderstanding, let us state clearly that the considerations given above do not in any way affect the validity and applicability of the Shannon concept of entropy as used in communication theory. The entropy as defined by Shannon is not a physical entropy but a dimensionless probability measure. The Boltzmann definition of physical entropy:

$$
S_{\text {Boltzmann }}=-k \log W=-k \sum_{i} f_{i} \log f_{i}
$$

is used by Shannon, but in the dimensionless form:

$$
\left(\frac{S_{\text {Boltzmann }}}{k}\right) \longrightarrow S_{\text {Shannon }}=-\log W=-\sum f_{i} \log f_{i}
$$

Clearly the "Shannon entropy" is a probability measure, and not a physical entropy, since it is dimensionless and the probabilities do not refer to the normalized phase space $(d p d q / h)$ of any quantum mechanical degrees of freedom.

2.

An Analysis of Szilard's Construction

\subsection{Introduction}

Szilard [8] in his famous paper of 1929 is credited with demonstrating that information is necessarily connected with physical entropy, so that one bit of information corresponds to the entropy $\geq k \log 2$. In our analysis of the nature of Boltmann's constant, in Section 1, above, we demonstrated that pure information-that is, idealized information-has no physical entropy associated with it. These two results are incompatible, and accordingly we need to investigate this problem, by examining both results critically, in the specific context of Szilard's construction. That is the purpose of this concluding section. 


\subsection{Resumé of Szilard's thought experiment}

In our description of Szilard's thought experiment [8], we follow closely Jauch and Barón's version [11]. They made only physically irrelevant changes in Szilard's experimental set-up to make the system more convenient for analysis.

In the experiment, a rigid, hollow, heat-permeable cylinder, closed at both ends, is used. It is fitted with a freely moveable piston with a hole large enough for the molecule to pass easily through it. The hole can be closed from the outside. All motions are considered reversible and frictionless. The cylinder is in contact with a very large heat reservoir to keep the temperature of the entire machine constant.

Within the cylinder is a gas consisting of a single molecule. At the beginning of the experiment, the piston is in the middle of the cylinder and its hole is open so that the molecule can move (almost) freely from one side of the piston to the other. The hole is then closed, trapping the molecule in one half of the cylinder.

The observer now determines the location of the molecule by a process called by Szilard 'Messung' (measurement). If it is found to the left of the piston, the observer attaches a weight to the piston with a string over a pulley so that the pressure is almost counterbalanced. He then moves the piston very slowly to the right, thereby raising the weight. When the piston reaches the end of the cylinder, the hole in the piston is opened and the piston is moved back to the middle of the cylinder, reversibly and without effect on the gas. At the end of this process the starting position has been reached, except that a certain amount of heat energy $Q$ from the heat reservoir has been transformed into potential energy $A$ of the weight lifted.

By repeating the process a large number of times (say, $N$ ), an arbitrarily large quantity of the heat energy $Q=N A$ from the reservoir is transformed into potential energy without any other change in the system. This violates the second principle of thermodynamics.

In order to 'save' the second law, Szilard assumes that the observation of the molecule, for determining in which half of the cylinder it is contained, is in principle connected with an exactly compensating increase of entropy of the observer. In this way, Szilard postulates his basic result as eq. (3) of Section 1, above.

\subsection{The Necessity for Observation}

The necessity for observation, in order to have Szilard's machine function, was questioned very early on by Popper [12] and by Jauch and Barón [11]. These authors pointed out that it was not necessary to decide which half of the machine the molecule was in, after the shutter was closed, since it was easy to allow the system to expanddoing external work-regardless of which half contained the molecule.

If this is correct, it brings Szilard's conclusion immediately into question, at its very foundation, since Szilard's whole point was to prove that "information" supplied the missing entropy in a cyclic process which would otherwise violate the second law of thermodynamics.

Like so many plausible considerations on Szilard's engine, their remark is neither true nor false, since Popper and Jauch-Barón have pre-supposed that classical physics suffices to discuss a one-molecule heat engine. As we will demonstrate conclusively, the use of quantum-mechanics is essential in Szilard's problem. 
Remark: The fact that quantum-mechanics is essential in discussing classical thermodynamics correctly is well known [5], [7], [8]. We have already cited the fact that the validity of the zeroth law [7] requires the classical dilemma on the stability of atoms to be resolved by quantum mechanics. Similarly, the Boltzmann definition of entropy required quantum mechanics to determine the unique cell size in phase space. Quantum mechanics also is required to justify the third law of thermodynamics [10].

Thus it is quite natural that Szilard's daring use of a one-molecule heat engine, using classical thermodynamics, cannot be correct without explicit consideration of quantum mechanics.

With no loss in generality, we can, however, confine Szilard's engine to one dimension, which greatly simplifies the manipulations. A single molecule in a onedimensional box is a standard quantum mechanical problem. The wave function $\Psi(x)$, has the boundary conditions:

$$
\Psi(0)=\Psi(a)=0
$$

and the wave functions are:

$$
\Psi=\sqrt{\frac{2}{a}} \sin \left(\frac{N \pi x}{a}\right), \quad N=1,2, \ldots,
$$

with eigenenergies:

$$
E_{N}=\frac{h^{2}}{2 M} \times\left(\frac{N \pi}{a}\right)^{2}
$$

Let us put this system into thermal equilibrium at the temperature $T$. Then:

$$
f_{(N)} \equiv \text { Probability of state } N=\frac{e^{-E_{N} / k T}}{\sum_{N=0}^{\infty} e^{-E_{N} / k T}}
$$

However, this one-molecule engine must be effectively classical-in order to carry out Szilard's argument-and this implies that the average quantum number, $N_{\text {ave }}$ is $N_{\text {ave }} \gg 1$ so that $k T$ itself is macroscopic, $\left(\approx \frac{1}{40} e . v\right.$.) with $T \approx 300 \mathrm{deg} K$. (Taking $M \approx m_{\text {electron }}$ and $a \approx 1 \mathrm{~cm}$, one finds $N_{\text {ave }} \approx 10^{7}$, so that the quantum mechanics is well described semi-classically.) In this limit, this one-dimensional semi-classical gas (of one molecule!) then obeys perfect-gas laws.

\subsection{Operation of the Quantum-Mechanical One-Molecule Engine}

Now let us examine the physical operations Szilard assumes it is possible to carry out on his heat engine.

(a) Operation of the Shutter:

The action of "closing the shutter" is to divide the space occupied by the "gas" into two equal parts. We take this to mean that at $x=a / 2$, all of the wave functions of the molecule must vanish:

$$
\text { shutter closed } \Rightarrow \Psi(x=a / 2)=0 \text {. }
$$


(b) What effect does closing the shutter have on the thermal wave function?

Clearly the shutter makes all of the admissible states vanish at $x=a / 2$. At first glance, this appears to mean that the quantum number $N$ goes in effect to $2 N$ (with the same $a$ ) - that is, the available volume for the one-molecule gas decreases by $1 / 2$ - but this is not correct.

In fact, for small $N$, we have:

$N=$ odd integer, $\Rightarrow$ large changes in the region centered at $x=a / 2$

$N=$ even integer $\Rightarrow$ no change at $x=a / 2$

For large $N$ :

$N=$ even integer, no change; $N=$ odd integer, very small change effectively only in the immediate vicinity of

$$
x=a / 2, \text { with size } \approx a / 2 N
$$

(Effectively, the wave function shifts to a linear combination principally of $N^{\prime}=$ $N \pm 1$.

Result: For a thermal wave function, closing the shutter makes a negligible (microscopic) change in the (macroscopic) average energy: $\Delta E / E \approx N_{\text {ave }}^{-1}, N_{\text {ave }} \gg 1$.

This result is exactly what one would expect for a classical gas (with $\approx$ Avogadro's number of molecules), that is, dividing a classical gas into two pieces does not in any way alter the energy or the entropy. Since the pressure on both sides of the shutter is the same, the shutter does not move (assuming that the system is so constructed that the shutter could move to extract energy).

We see that Szilard's one-molecule gas-at a macroscopic temperature - is effectively unchanged in energy or entropy if we insert a shutter dividing the system into two parts.

Why then does Szilard claim that-after the shutter is closed-the "side containing the molecule" is at a differential pressure so that work can be extracted? The answer is that Szilard assumes that after the shutter is closed we then observe the molecule to be, say, on the left hand side, and subsequently, we extract heat from this side by expansion.

This assumption which Szilard has made seems very plausible. After all, in our one-molecule quantum mechanical "gas," the molecule is certain to be measured on the right hand side or the left hand side with probability $1 / 2$ for either.

But matters are not quite this simple. To observe the molecule on one side (LHS, say), means that the resulting quantum state has zero probability for all states where the wave function is non-zero on the right hand side.

That is: observation makes a profound difference in the thermal wave function: observation implies

$$
\Psi_{\text {thermal }} \rightarrow \begin{cases}\Psi_{\text {new }}, & 0 \leq x \leq a / 2 \\ 0, & a / 2 \leq x \leq a\end{cases}
$$

The new 'thermal' wave function depends critically on the measurement. However one chooses to define this "observation," it is clear that the new state is, energetically, macroscopically much larger. Qualitatively, the observation has compressed 
"the one-molecule gas" to exactly half the volume. This compression-be it adiabatic, isothermal or some variant thereof - then permits energy to be extracted by expansion, since work has been done on the system, increasing its energy.

Clearly there is no violation whatever of the second law (any entropy change came from the mechanics of the way the "observation" was made). It is equally clear that no energy can be extracted that wasn't put into the system by the preparation ("observation") of the new state.

\subsection{Further Analysis}

To be quite clear let us analyze this sequence of events further. We have seen that inserting the shutter in the system, dividing the "gas" into two parts makes a negligible change in the energy and entropy of the system (just as it would classically for a macroscopic gas with an idealized (vanishingly thin) partition). Until the "gas" is observed to be confined to one side or the other, the one-molecule gas still has the same physical space available. This is non-intuitive to be sure, but quantum mechanics is unequivocal on this point.

This analysis shows that the objection of Popper and Jauch and Barón-that the Szilard engine could extract energy without requiring any observation-is clearly wrong. Without observation the "one-molecule" gas (with a closed shutter dividing space into two parts) is essentially unchanged, the molecule still has both sides available for its thermal wave function. This situation is precisely the analog of the familiar two-slit "paradox" of quantum mechanics: the photon goes through both slits (unless observed to do otherwise).

The act of observation is not a simple procedure of deciding between two alternatives. The effect of observation prepares the system in a state $\Psi_{\text {new }}$ as given by eq. (12), but the occupation numbers of the denumerably infinite number of possible eigenstates in the confined space must be determined. (More precisely one doesn't even have a superposition of eigenstates, since the system is surely going to be overwhelmingly likely to be statistical, that is, incoherent with random phases.)

The physical entropy change caused by the observation is by no means limited to a simple choice of one of two alternatives, that is, $k \log 2$. The entropy change is given by quantum mechanics as:

$$
\Delta S / k=\sum_{N} f_{(N)}^{\text {initial }} \log f_{(N)}^{\text {initial }}-\sum_{N} f_{(N)}^{\text {initial }} \log f_{(N)}^{\text {initial }}
$$

where $f_{(N)} \cdots$ are the level occupation probabilities. It is not obvious that this entropy change is necessarily microscopic $(\Delta S / k \approx 1)$, since for $N \approx 10^{7}, \Delta S$ might conceivably be macroscopic.

Out of the myriad of possible observations, let us select one which recommends itself as the simplest. Let us choose to make our observation adiabatically. We can certainly do this, by imagining that instead of inserting the shutter and then observing, we instead slowly compress the gas moving the divider (shutter) from one end to the middle. (Of course, to be adiabatic one must isolate the one-molecule system from the heat bath temporarily.)

Quantum mechanically, the word adiabatic has the meaning that the occupation probabilities $f_{N} \cdots$ are to be unchanged in the process. The energies of course do 
change slowly, by a factor of 4 when the system is slowly compressed by a factor of 2. To maintain the adiabaticity condition, eq. (12) shows that the temperature must also increase slowly, in concert with the slow increase in energy, ending up at a final temperature a factor of 4 larger than initially.

The final state achieved by the adiabatic process can be seen to be a fully acceptable "observed" state just as if it were to be achieved by closing the shutter and "observing" (even though it is most improbable to expect to find such an adiabatic state in this latter way!).

The next step in Szilard's heat engine cycle is to expand this state (possibly even adiabatically) and extract energy. This can certainly be done, since the new state has a much higher temperature, but of course we are not going to profit by this extracted energy!

The really essential point, however, is that we can achieve, in principle, a complete cycle of the Szilard heat engine without any entropy change whatsoever. Quite clearly the physical entropy change eq. (13) has nothing whatever to do with $k \log 2$.

Let us return to the original question: Did Szilard demonstrate that "information" is associated with physical entropy? In particular, did he prove that the "information" (in running one cycle of the Szilard engine) supplies physical entropy $k \log 2$ ?

Consider the previous analysis. The Szilard "information" was one of two alternatives. Hence, the Shannon, dimensionless, information is indeed $\log 2$. By contrast, the physical information amounts to defining the new state, eq. (12). The resulting energy change (from "preparing" (or, equivalently, "observing")) the new "thermal" state is macroscopic ( $\Delta E \approx \frac{1}{10} e . v$.), whereas the entropy change depends critically on how the new state is prepared. One can even, in principle, make the new state by an adiabatic process (as above) so that the change in entropy is zero.

There is simply no way to compare these two "entropies". The point is that the physical entropy change was determined completely by the physical operations on the system. By contrast, the Shannon entropy is a priori, independent of method of measurement. After making the observation, the Szilard engine can certainly be used to extract energy. This is clear since the "gas" is compressed (by the observation) and can be expanded to extract energy. Where does this energy come from? It is clearly energy put into the gas by the act of observation. There is accordingly no violation of either the first or second law of thermodynamics.

In our view, these considerations make it very clear that information per se is a subjective, idealized, concept independent of physical entropy. Physical entropy depends on physical objects and physical interations, and any entropy change due to observations is entirely due to the entropy changes in the physical apparatus (including the system observed) in carrying out the observations.

3.

Acknowledgement

This work was supported in part by grant DOE-ER-40757-062 of the Center for Particle Physics and is preprint CPP-95-2.

4.

References 
1. E. Richard Cohen and Barry N. Taylor, Physics Today, BG 9-13, (1994).

2. S. Weinberg, The Constants of Physics, Philos. Trans. Roy. Soc. Lond. A 310, (1983) 249-252.

3. G. Veneziano, CERN-TH.6725/92 (1992).

4. P. W. Bridgman, Dimensional Analysis (Yale University Press, New Haven, (1931)); B. Ellis, Basic Concepts of Measurement (Cambridge University Press, Cambridge, (1966)); L.B. Okun, Sov. Phys. Usp. 34 (1991) 818; L.B. Okun, Particle physics: the quest for the substance of substance (Harwood Academic Publishers (1985)).

5. Max Born, Natural Philosophy of Cause and Chance, Waynflete Lectures delivered in the College of St. Mary Madalen, Oxford; (Oxford at the Clarendon Press 1948).

6. H. Koppe and A. Huber, Zum Problem der ab-initio Berechnung der Boltzmannkonstante, in Quanten und Felder, Phyikalische and philosophische Betrachtungen zum 70. Geburstag von Werner Heisenberg, ed.by H.P. Dürr, F. Vieweg und Sohn, (Braunschweig, B.R.D.).

7. Laszlo Tisza, Generalized Thermodynamics, (Massachusetts Institute of Technology Press, Cambridge, Massachusetts 1966).

8. L. Szilard, Z. f. Physik 53, 840-856 (1929).

9. H.S. Leff and A.F. Rex, Maxwell's Demon Entropy, Information, Computing, (Adam Hilger, Bristol 1990).

10. Enrico Fermi, Thermodynamics, (Prentice-Hall, New York 1937).

11. J.M. Jauch and J.G. Barón, Helv. Phys. Acta 45, 220-232 (1972).

12. K. Popper, Brit. J. Phil. Sci. 8, 151-155 (1957).

\section{DISCLAIMER}

This report was prepared as an account of work sponsored by an agency of the United States Government. Neither the United States Government nor any agency thereof, nor any of their employees, makes any warranty, express or implied, or assumes any legal liability or responsibility for the accuracy, completeness, or usefulness of any information, apparatus, product, or process disclosed, or represents that its use would not infringe privately owned rights. Reference herein to any specific commercial product, process, or service by trade name, trademark, manufacturer, or otherwise does not necessarily constitute or imply its endorsement, recommendation, or favoring by the United States Government or any agency thereof. The views and opinions of authors expressed herein do not necessarily state or reflect those of the United States Government or any agency thereof. 
